\title{
Imaging "Pinwheel" nebulae with optical long-baseline interferometry
}

\author{
Florentin Millour ${ }^{1,2}$, Thomas Driebe ${ }^{1,3}$, Jose H. Groh ${ }^{1}$, Olivier \\ Chesneau $^{2}$, Gerd Weigelt ${ }^{1}$, Adriane Liermann ${ }^{1}$ and Anthony \\ Meilland $^{1}$ \\ ${ }^{1}$ Max-Planck Institute for Radioastronomy, auf dem Hügel 69, 53121 Bonn, Germany \\ email: fmillour@oca.eu \\ ${ }^{2}$ Observatoire de la côte d'Azur, Bd de l'Observatoire, 06304 Nice, France \\ ${ }^{3}$ German Aerospace Center (DLR), Königswinterer Str. 522-524, 53227 Bonn, Germany
}

\begin{abstract}
Dusty Wolf-Rayet stars are few but remarkable in terms of dust production rates (up to $\left.\dot{\mathrm{M}}=10^{-6} \mathrm{M}_{\odot} / \mathrm{yr}\right)$. Infrared excesses associated to mass-loss are found in the sub-types WC8 and WC9. Few WC9d stars are hosting a "pinwheel" nebula, indirect evidence of a companion star around the primary. While few other WC9d stars have a dust shell which has been barely resolved so far, the available angular resolution offered by single telescopes is insufficient to confirm if they also host "pinwheel" nebulae or not. In this article, we present the possible detection of such nebula around the star WR 118. We discuss about the potential of interferometry to image more "pinwheel" nebulae around other WC9d stars.
\end{abstract}

Keywords. techniques: high angular resolution, techniques: interferometric, binaries (including multiple): close, stars: individual (WR 118), stars: winds, outflows, stars: Wolf-Rayet

\section{WR 118}

In 2008, we observed the dusty Wolf-Rayet (WR) star WR 118 using the Astronomical Beam Recombiner (AMBER, Petrov et al. 2007), at the focus of the Very Large Telescope Interferometer (VLTI). WR 118 had already been observed using speckle interferometry, on the BTA 6m telescope, in Russia (Yudin et al. 2001). The AMBER visibilities and closure phases were acquired at spatial frequencies up to 5 times larger than the previous speckle observations.

We clearly resolved the system with AMBER, with visibilities decreasing up to $\approx 55$ cycles/arc-second, and increasing again above. Such a visibility behavior is typical of an object containing a sharp edge in its intensity distribution. In addition, the closure phase, measured at three different moments during the night, is clearly non-equal to zero, meaning that WR 118 's dusty nebula is asymmetric.

We modelled WR 118's dusty nebula with several geometrical models, including a clumpy spherical wind and a "pinwheel" nebula. This last model provides a physical description of the system and best match the observed data. Therefore, we concluded that WR 118 probably hosts a "pinwheel" nebula, detected for the first time using longbaseline interferometry (Millour et al. 2009a).

\section{Preparing future observations}

Repeating the AMBER observations on WR 118 to confirm its "pinwheel" nature is the next step in this research program. One step forward is to assess the feasibility of imaging such targets with the current and future capabilities of the VLTI. For that, 

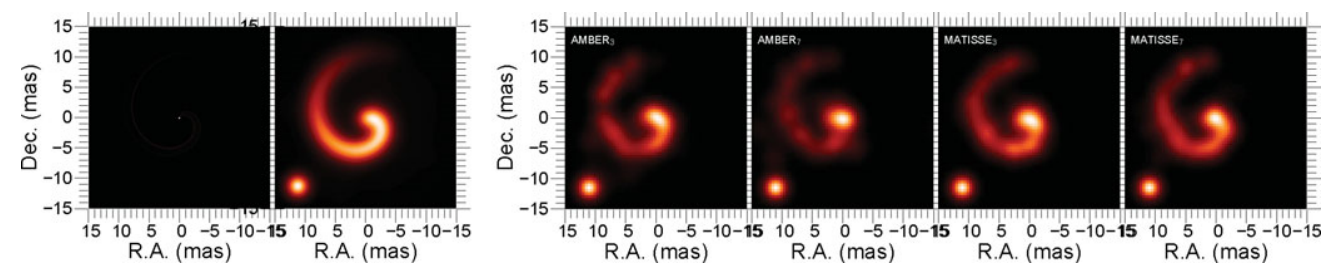

Figure 1. Simulations of aperture synthesis of "pinwheel" nebulae, using the parameters from WR 118. From left to right: the model used convolved with a beam equivalent to a $130 \mathrm{~m}$ diameter telescope, synthesised images for AMBER observations during 3 and 7 nights, and synthesised images for MATISSE observations during 3 and 7 nights.

we perform simulations of the current VLTI instrument AMBER (K-band, 2.2 microns) and of the second generation planned VLTI instrument MATISSE (L-band, 3.5 microns) observations. We start from the model of WR 118 found previously and simulate a realistic observation (typical $\mathrm{V}^{2}$ errors of $5 \%$ ) with different baselines and one measurement per hour. Then, we use the MIRA software (Thiébaut 2008) to reconstruct an image of the target from these simulations. Four cases are simulated here:

- AMBER observations during 3 nights with 3 telescopes configurations,

- AMBER observations during 7 nights with 7 telescopes configurations,

- MATISSE observations during 3 nights with 3 telescopes configurations,

- MATISSE observations during 7 nights with 7 telescopes configurations.

As expected, we find that AMBER provides some imaging capabilities, even using as few as 3 nights of observation. One can recognize in the reconstructed image all the features from the original image (Fig. 1). However, as experienced on real datasets (Millour et al. 2009b), the relative flux of the different features is not well constrained, because of the lack of $66 \%$ of phase information. Increasing, by more than a factor 2 , the number of nights used, only marginally improves the quality of the image reconstruction.

On the other hand, MATISSE simulations show a better agreement on the fluxes of the different features, even using "only" three nights of observation. We also note that the loss in angular resolution from the K-band and L-band do not apparently affect the quality of the image reconstruction.

\section{Conclusion}

We showed here that long-baseline interferometry is now clearly mature to confirm the brightest suspected "pinwheel" nebulae, using the current abilities of AMBER on the VLTI. In addition, we also showed that AMBER will be able to synthethise an image, where the "pinwheel" nebula would be qualitatively recognized, using at least three nights of observing time. The use of the second generation MATISSE instrument will permit to have more realistic flux measurements in the different parts of the image, with the same amount of observing time.

\section{References}

Millour, F., Driebe, T., Chesneau, O., Groh, J. H. et al. 2009a, A\&AA, 506, L49

Millour, F., Chesneau, O., Borges Fernandes, M., Meilland, A. et al. 2009b, A\&AA, 507, 317

Petrov, R. G., Malbet, F., Weigelt, G., Antonelli, P. et al. 2007, A\&\&A, 464, 1-12

Thiébaut, E. 2008, in: M. Schöller, W. C. Danchi, \& F. Delplancke (eds.), Optical and Infrared Interferometry, SPIE Conference Series 7013, p. 43

Yudin, B., Balega, Y., Blöcker, T., Hofmann, K.-H. et al. 2001, A\& A, 379, 229 